\title{
The essential spectrum of the volume integral operator in electromagnetic scattering by a homogeneous body
}

\author{
Martin Costabel, Eric Darrigrand, Hamdi Sakly \\ IRMAR, Université de Rennes1, Campus de Beaulieu, 35042 Rennes \\ Received $* * * * *$; accepted after revision +++++ \\ Presented by
}

\begin{abstract}
We study the strongly singular volume integral operator that describes the scattering of time-harmonic electromagnetic waves. For the case of piecewise constant material coefficients and smooth interfaces, we determine the essential spectrum. We show that it is a finite set and that the operator is Fredholm of index zero in $\mathbf{H}(\mathrm{curl})$ if and only if the relative permeability and permittivity are both different from 0 and -1 .
\end{abstract}

\section{Résumé}

Le spectre essentiel de l'opérateur intégral volumique en diffraction électromagnétique par un corps homogène. Nous étudions le spectre essentiel de l'opérateur intégral volumique fortement singulier décrivant la diffraction d'ondes électromagnétiques. Dans le cas de coefficients constants par morceaux et pour une interface régulière nous démontrons qu'il est fini et que l'opérateur intégral est Fredholm d'indice zéro dans $\mathbf{H}$ (curl) si et seulement si les perméabilité et permittivité relatives sont différentes de 0 et de -1 .

\section{Version française abrégée}

Nous nous intéressons à la diffraction d'ondes électromagnétiques harmoniques en temps par $\Omega$, un domaine borné de $\mathbb{R}^{3}$. Lorsque $\Omega$ est un objet pénétrable, le problème est régi par les équations de Maxwell (4) satisfaites dans $\mathbb{R}^{3}$ au sens des distributions, complétées par une condition de rayonnement à l'infini.

Afin de ramener le problème à un domaine borné, nous considérons sa formulation par une équation intégrale volumique (VIE) (3) dans laquelle apparaissent deux opérateurs fortement singuliers $A_{k}$ et $B_{k}$,

Email addresses: martin.costabel@univ-rennes1.fr (Martin Costabel), eric.darrigrand-lacarrieu@univ-rennes1.fr (Eric Darrigrand), hamdi.sakly@univ-rennes1.fr (Hamdi Sakly). 
voir (2). Cette équation, aussi appelée équation de Lippmann-Schwinger [3] est couramment utilisée en pratique pour des méthodes numériques, et la question de son spectre essentiel a été soulevée dans la littérature $[1,2,10]$. La connaissance du spectre essentiel est importante pour deux raisons : La convergence d'algorithmes itératifs en dépend, et l'essor de métamatériaux remet en question les hypothèses "classiques" qui garantissent que le modèle mathématique soit bien posé.

Nous supposons ici que les coefficients $\varepsilon$ (permittivité électrique) et $\mu$ (perméabilité magnétique) prennent des valeurs constantes dans $\Omega$ et dans son complément, voir (1). C'est la situation opposée à celle de coefficients continus dans tout l'espace pour laquelle il est connu que la VIE est équivalente à une équation de seconde espèce faiblement singulière [3], le spectre essentiel étant réduit au seul point $\{1\}$. Pour le cas de coefficients discontinus, une conjecture basée sur des observations numériques a été énoncée $[2,10]$, mais nous démontrons qu'elle n'est pas vraie. Le cas d'un problème magnétostatique a déjà été étudié en 1985 [6]. Dans notre formalisme, ceci correspond au cas électrique analysé dans la section 3. Dans les travaux $[7,8]$, la VIE est étudiée pour des coefficients plus généraux, dans $\mathbf{H}(\operatorname{curl}, \Omega)$ et dans $\mathbf{L}^{2}(\Omega)$, sans pourtant répondre à la question du spectre essentiel. Les deux opérateurs $A_{k}$ et $B_{k}$ montrent des propriétés assez différentes : L'opérateur $A_{k}$ est à une perturbation compacte près un opérateur autoadjoint borné dans $\mathbf{L}^{2}(\Omega)$ qui permet une description simple par des sous-espaces invariants. La partie "pertinente" de son spectre essentiel est reliée à un opérateur intégral de frontière scalaire qui est bien connu de l'analyse harmonique. L'opérateur $B_{k}$ ne permet pas d'extension de $\mathbf{H}(\mathrm{curl})$ à $\mathbf{L}^{2}$, et son spectre essentiel est déterminé par un système d'opérateurs intégraux de frontière. Ce système (7) contient des opérateurs que l'on connaît de la méthode des équations intégrales de frontière pour le problème de diffraction, sans pour autant provenir de cette méthode. Malgré cette différence, pour un bord régulier les spectres essentiels des opérateurs $A_{k}$ et $B_{k}$ sont confondus et égaux à $\left\{0, \frac{1}{2}, 1\right\}$. Nous avons le résultat suivant qui montre que $A_{k}$ et $B_{k}$ ont des rôles symétriques mais sont en quelque sorte orthogonaux.

Theorem 0.1 Soit $\Omega$ un domaine borné de classe $C^{\infty}$ et $\eta, \nu \in \mathbb{C}$. Alors le spectre essentiel de l'opérateur $\eta A_{k}+\nu B_{k}$ est l'ensemble $\left\{0, \frac{\eta}{2}, \eta, \frac{\nu}{2}, \nu\right\}$. En conséquence, la VIE est Fredholm d'indice zéro si et seulement si $\eta, \nu \notin\{1,2\}$, et le problème de diffraction est elliptique si et seulement si les perméabilité et permittivité relatives sont différentes de 0 et de -1 .

\section{Introduction}

We consider the scattering of time-harmonic electromagnetic waves by a penetrable object. The mathematical model is given by the Maxwell equations depending on three parameters: The frequency $\omega \in \mathbb{R}$, the electric permittivity $\varepsilon$ and the magnetic permeability $\mu$. Assuming the medium is isotropic, both $\varepsilon$ and $\mu$ are scalar functions defined on $\mathbb{R}^{3}$. If $\varepsilon$ and $\mu$ are constant outside a bounded domain $\Omega$, then the scattering problem can equivalently be described by an integral equation in $\Omega$, the Lippmann-Schwinger equation of electromagnetic scattering [3, Chap. 9].

This integral equation, sometimes simply called "volume integral equation" (VIE) of electromagnetic scattering, has been intensively used by physicists for numerical calculations, see [1] and the references therein. It is a strongly singular integral equation on the domain $\Omega$, which can, if $\varepsilon$ and $\mu$ are smooth on the whole space, be rewritten as a weakly singular Fredholm integral equation of the second kind on $\Omega$. In this form, it is used in [3] as a tool in the existence proof for the dielectric scattering problem.

When $\varepsilon$ and $\mu$ are discontinuous on the surface of the scatterer, which is the situation most commonly encountered in the applications, this reduction to a weakly singular Fredholm integral equation is not possible, and the integral operator is expected to have a non-trivial essential spectrum. A complete mathematical analysis of this case does not yet seem to be available in the literature. Partial results have been obtained in $[6,5,7,8]$. In [10], the importance of knowing the spectrum of the integral operator for the 
analysis of numerical solution methods is emphasized, but only numerical evidence for the extent of the essential spectrum is presented. In [2], only the case of everywhere continuous coefficients is considered. In both papers $[2,10]$, however, conjectures about the essential spectrum are offered for the case of discontinuous coefficients. In the simple case where $\mu$ is constant and $\varepsilon$ is piecewise constant, $\varepsilon=\varepsilon_{0}$ in $\mathbb{R}^{3} \backslash \Omega, \varepsilon=\varepsilon_{r} \varepsilon_{0}$ in $\Omega$, the conjectures mean that the line segment connecting 1 and $\varepsilon_{r}$ is contained in the essential spectrum. It follows from the results of this note that this is not correct: If the boundary of $\Omega$ is smooth, then the essential spectrum consists of the endpoints and the midpoint of that segment. For the case of a Lipschitz boundary, one can already deduce from the results of [6] that the endpoints of the segment are isolated points of the essential spectrum.

In this note, we consider the case where both $\varepsilon$ and $\mu$ are piecewise constant,

$$
\varepsilon=\varepsilon_{0}, \mu=\mu_{0} \quad \text { in } \mathbb{R}^{3} \backslash \Omega ; \quad \varepsilon=\varepsilon_{r} \varepsilon_{0}, \mu=\mu_{r} \mu_{0} \quad \text { in } \Omega .
$$

Here the vacuum permittivity $\varepsilon_{0}$ and permeability $\mu_{0}$ are positive real constants, and the relative permittivity $\varepsilon_{r}$ and permeability $\mu_{r}$ can be arbitrary complex numbers. With a positive frequency $\omega$ and wave number $k=\omega \sqrt{\varepsilon_{0} \mu_{0}}$ we define the fundamental solution of the Helmholtz equation

$$
g_{k}(x)=\frac{e^{i k|x|}}{4 \pi|x|}
$$

and the integral operators $A_{k}$ and $B_{k}$

$$
\begin{aligned}
& A_{k} u(x)=-\nabla \operatorname{div} \int_{\Omega} g_{k}(x-y) u(y) d y-k^{2} \int_{\Omega} g_{k}(x-y) u(y) d y \\
& B_{k} u(x)=\operatorname{curl} \int_{\Omega} g_{k}(x-y) \operatorname{curl} u(y) d y .
\end{aligned}
$$

With the electric and magnetic contrast $\eta=1-\varepsilon_{r}$ and $\nu=1-1 / \mu_{r}$, the VIE can then be written as

$$
u(x)-\eta A_{k} u(x)-\nu B_{k} u(x)=u^{0}(x) \quad(x \in \Omega) .
$$

The main result of this note, see Theorem 5.1, implies for the integral equation (3) the following result. Theorem 1.1 Let $\Omega \subset \mathbb{R}^{3}$ be a bounded domain with $C^{\infty}$ boundary and $\eta, \nu \in \mathbb{C}$. Then the operator $1-\mathcal{A}_{k}^{\eta, \nu}: u \mapsto u-\eta A_{k} u-\nu B_{k} u$ is a Fredholm operator of index zero in $\mathbf{H}(\mathrm{curl}, \Omega)$ if and only if $\eta \notin\{1,2\}$ and $\nu \notin\{1,2\}$. In addition, if $\nu=0$, then the operator $1-\mathcal{A}_{k}^{\eta, 0}: u \mapsto u-\eta A_{k} u$ is a Fredholm operator of index zero in $\mathbf{L}^{2}(\Omega)$ if and only if $\eta \notin\{1,2\}$.

By equivalence with the time-harmonic Maxwell scattering problem (4), we see that the latter is an elliptic transmission problem if and only if the coefficients $\varepsilon_{r}$ and $\mu_{r}$ are different from 0 and -1 .

\section{Scattering of time-harmonic electromagnetic waves by a homogeneous penetrable object and the electromagnetic Lippmann-Schwinger equation}

We assume that $\Omega \subset \mathbb{R}^{3}$ is a bounded domain with a Lipschitz boundary. For some of the results below we will assume $C^{\infty}$ regularity for $\partial \Omega$. With positive numbers $\varepsilon_{0}$ and $\mu_{0}$ and complex numbers $\varepsilon_{r}$ and $\mu_{r}$, we define the piecewise constant functions $\varepsilon$ and $\mu$ by (1). The time-harmonic electromagnetic scattering problem for an incident wave $\left(E^{0}, H^{0}\right)$ and a positive frequency $\omega$ is described by the Maxwell equations

$$
\operatorname{curl} E=i \omega \mu H ; \quad \operatorname{curl} H=-i \omega \varepsilon E+J
$$

valid in $\mathbb{R}^{3}$ in the distributional sense, completed by the standard Silver-Müller radiation condition for the scattered field $\left(E-E^{0}, H-H^{0}\right)$. The incident fields satisfy the free-space Maxwell equations

$$
\operatorname{curl} E^{0}=i \omega \mu_{0} H^{0} ; \quad \operatorname{curl} H^{0}=-i \omega \varepsilon_{0} E^{0}+J
$$


in $\mathbb{R}^{3}$. This fixes the current density $J$, which we assume to be of compact support and divergence free, implying in particular $\operatorname{div} E^{0}=0$ and $\operatorname{div} \varepsilon E=0$. It is natural to assume that all fields are locally square integrable and in view of (4) therefore that $E$ and $H$ belong to $\mathbf{H}_{\text {loc }}\left(\operatorname{curl}, \mathbb{R}^{3}\right)$. In this framework, it is known that the problem (4) is equivalent to the volume integral equation (3) considered in $\mathbf{H}(\operatorname{curl}, \Omega)$ [7]. We formulate here the equivalence with the electric field as the principal unknown. A symmetric formulation for the magnetic field is possible, which is the choice in $[7,8]$.

Proposition 2.1 Let $E, H$ solve (4) and let $u \in \mathbf{H}(\operatorname{curl}, \Omega)$ be the restriction of $E$ to $\Omega$. Then u solves the VIE (3) with $u^{0}=E^{0}$. Conversely, let $u \in \mathbf{H}(\operatorname{curl}, \Omega)$ solve the VIE (3) with $u^{0}=E^{0}$. Extend $u$ to $x \in \mathbb{R}^{3}$ by the same formula (3) and define $E=u, H=\operatorname{curl} u /(i \omega \mu)$. Then $E, H$ solve the scattering problem (4).

As a partial answer to the problem of solvability of the VIE (3), we shall characterize here the set of $\eta, \nu \in \mathbb{C}$ for which the strongly singular integral equation (3) is Fredholm of index zero in $\mathbf{H}(\operatorname{curl}, \Omega)$, or equivalently, for which the number 1 does not belong to the essential spectrum $\sigma_{e}\left(\mathcal{A}_{k}^{\eta, \nu}\right)$.

Besides the natural function space $\mathbf{H}(\operatorname{curl}, \Omega)$, it is clearly also interesting to consider the operator in $\mathbf{L}^{2}(\Omega)$. We use standard notation for Sobolev spaces, and we denote spaces of vector-valued functions by boldface letters.

\section{The essential spectrum of the electric volume integral operator}

In the case of a purely dielectric obstacle, we have $\nu=0$, and the VIE is reduced to the equation $u-\eta A_{k} u=u^{0}$. It is easy to see that $A_{k}$ is a bounded operator in $\mathbf{H}(\operatorname{curl}, \Omega)$ and that $\operatorname{div} A_{k} u=\operatorname{div} u$. This implies the following result; details can be found in [5].

Theorem 3.1 The operator $A_{k}$ can be extended from $\mathbf{H}(\operatorname{curl}, \Omega)$ to $\mathbf{L}^{2}(\Omega)$ as a bounded operator having $\mathbf{H}(\operatorname{curl}, \Omega)$ and $\mathbf{H}(\operatorname{div}, \Omega)$ as invariant subspaces. For $u_{0} \in \mathbf{H}(\operatorname{curl}, \Omega) \cap \mathbf{H}(\operatorname{div}, \Omega)$, the VIE with $\nu=0$ has the same solutions, whether considered in $\mathbf{L}^{2}(\Omega)$ or in $\mathbf{H}(\operatorname{curl}, \Omega)$ or in $\mathbf{H}(\operatorname{div}, \Omega)$.

It is easy to see that $A_{k}-A_{0}$ maps $\mathbf{L}^{2}(\Omega)$ boundedly to $H^{2}(\Omega)$, which implies that $A_{k}-A_{0}$ is a compact operator both in $\mathbf{L}^{2}(\Omega)$ and in $\mathbf{H}(\operatorname{curl}, \Omega)$. For the determination of the essential spectrum of the operator $A_{k}$, it is therefore sufficient to study the essential spectrum of the operator $A_{0}$. We give a slightly more precise version of a result already shown in [6]. It is based on the orthogonal decomposition

$$
\mathbf{L}^{2}(\Omega)=\nabla \mathrm{H}_{0}^{1}(\Omega) \oplus \mathbf{H}_{0}(\operatorname{div} 0, \Omega) \oplus \mathbf{W}
$$

where $\mathbf{H}_{0}(\operatorname{div} 0, \Omega)$ is the space of divergence-free $\mathrm{L}^{2}$ vector fields with vanishing normal trace, and $\mathbf{W}$ is the space of gradients of harmonic $\mathrm{H}^{1}$ vector fields. The normal trace $\gamma_{n}$ defines an isomorphism between W and the space $\mathrm{H}_{*}^{-\frac{1}{2}}(\partial \Omega)$ of $\mathrm{H}^{-\frac{1}{2}}$ functions of vanishing mean value on the boundary.

Theorem 3.2 The operator $A_{0}$ is bounded and selfadjoint on $\mathbf{L}^{2}(\Omega)$ with $\nabla \mathrm{H}_{0}^{1}(\Omega), \mathbf{H}_{0}(\operatorname{div} 0, \Omega)$ and $\mathbf{W}$ as invariant subspaces. On $\nabla \mathrm{H}_{0}^{1}(\Omega): A_{0} u=u$, on $\mathbf{H}_{0}(\operatorname{div} 0, \Omega): A_{0} u=0$, and on $\mathbf{W}: \gamma_{n} A_{0}=\left(\frac{1}{2}+K_{0}^{\prime}\right) \gamma_{n}$, where $K_{0}^{\prime}$ is the operator of the normal derivative of the harmonic single layer potential.

It is known that $\frac{1}{2}+K_{0}^{\prime}$ is a positive selfadjoint operator in $\mathrm{H}_{*}^{-\frac{1}{2}}(\partial \Omega)$ that differs at most by an operator of finite rank from a contraction. This follows from estimates similar to those in [6], see [4]. If $\partial \Omega$ is smooth, it is well known that $K_{0}^{\prime}$ is compact in $\mathrm{H}_{*}^{-\frac{1}{2}}(\partial \Omega)$.

Corollary 3.3 The essential spectrum of $A_{k}$ is the same in $\mathbf{L}^{2}(\Omega)$ and in $\mathbf{H}(\operatorname{curl}, \Omega)$. There exist $0<$ $\lambda \leq \Lambda<1$ such that $\sigma_{e}\left(A_{k}\right) \subset\{0,1\} \cup[\lambda, \Lambda]$. If $\partial \Omega$ is smooth, then $\lambda=\Lambda=\frac{1}{2}$, so that $\sigma_{e}\left(A_{k}\right)=\left\{0, \frac{1}{2}, 1\right\}$. 


\section{The essential spectrum of the magnetic volume integral operator}

If only $\mu$ is discontinuous, we have $\eta=0$, and the VIE is reduced to the equation $u-\nu B_{k} u=u^{0}$. It is easy to see that $B_{k}$ is a bounded operator in $\mathbf{H}(\operatorname{curl}, \Omega)$ and that $\operatorname{div} B_{k} u=0$. The direct parallel with $A_{k}$ ends here, however. By partial integration, we can rewrite $B_{k}$ as follows

$$
B_{k} u(x)=\operatorname{curlcurl} \int_{\Omega} g_{k}(x-y) u(y) d y+\operatorname{curl} S_{k} \gamma_{\times} u(x)=-A_{k} u(x)+u(x)+\operatorname{curl} S_{k} \gamma_{\times} u(x) .
$$

Here $\gamma_{\times}: \mathbf{H}(\operatorname{curl}, \Omega) \rightarrow \mathbf{H}^{-\frac{1}{2}}\left(\operatorname{div}_{\top}, \partial \Omega\right)$ is the tangential trace operator that coincides with $u \mapsto \gamma_{\times} u=$ $n \times u$ for smooth functions. By $S_{k}$ we denote the single layer potential, which maps $\mathrm{H}^{-\frac{1}{2}}(\partial \Omega)$ to $\mathrm{H}^{1}(\Omega)$.

The last term in (5) shows that $B_{k}$ cannot be extended from $\mathbf{H}(\operatorname{curl}, \Omega)$ to $\mathbf{L}^{2}(\Omega)$ as a bounded operator. If, however, we restrict $B_{k}$ to $\mathbf{H}_{0}(\operatorname{curl}, \Omega)$, this last term is absent, and we can extend this restriction to a bounded selfadjoint operator $B_{k}^{0}$ on $\mathbf{L}^{2}(\Omega)$ that satisfies $B_{k}^{0}=1-A_{k}$. From Corollary 3.3 we know the essential spectrum of $B_{k}^{0}$. For a smooth domain we have $\sigma_{e}\left(B_{k}^{0}\right)=\left\{0, \frac{1}{2}, 1\right\}$. In order to determine $\sigma_{e}\left(B_{k}\right)$, we first notice that $B_{k}=0$ on $\nabla \mathbf{H}^{1}(\Omega)$. If we define $\mathbf{X}=\mathbf{H}(\operatorname{curl}, \Omega) \cap \mathbf{H}(\operatorname{div} 0, \Omega)$, we see that in the orthogonal sum $\mathbf{H}(\operatorname{curl}, \Omega)=\nabla \mathbf{H}_{0}^{1}(\Omega) \oplus \mathbf{X}$, both terms are invariant under $B_{k}$, so that it suffices to consider $B_{k}$, and then $B_{0}$, in $\mathbf{X}$. We use integration by parts in (5) to obtain

$$
B_{0} u(x)=u(x)+\nabla \int_{\Omega} g_{0}(x-y) \operatorname{div} u(y) d y-\nabla S_{0} \gamma_{n} u(x)+\operatorname{curl} S_{0} \gamma_{\times} u(x) .
$$

For $u \in \mathbf{X}$ this means $B_{0} u=u-\nabla S_{0} \gamma_{n} u+\operatorname{curl} S_{0} \gamma_{\times} u$. Now we use the following elementary lemma.

Lemma 4.1 Let $X$ and $Y$ be vector spaces and $S: Y \rightarrow X$ and $T: X \rightarrow Y$ linear operators. Then for $\lambda \neq 0, T$ induces isomorphisms from $\operatorname{ker}(\lambda-S T)$ to $\operatorname{ker}(\lambda-T S)$ and from $X /(\lambda-S T) X$ to $Y /(\lambda-T S) Y$. In particular, $\lambda-S T$ is Fredholm of index 0 in $X$ if and only if $\lambda-T S$ is Fredholm of index 0 in $Y$. We apply this to the situation $X=\mathbf{X}, Y=\mathbf{Y}=\mathrm{H}^{-\frac{1}{2}}(\partial \Omega) \times \mathbf{H}^{-\frac{1}{2}}(\operatorname{div} \uparrow, \partial \Omega), T=\left(\gamma_{n}, \gamma_{\times}\right), S(v, w)=$ $-\nabla S_{0} v+\operatorname{curl} S_{0} w$. The result is that for $\lambda \neq 1, \sigma_{e}\left(B_{0}\right)=\sigma_{e}\left(\widehat{B}_{0}\right)$, where $\widehat{B}_{0}$ is the system of boundary integral operators in the space $\mathbf{Y}$

$$
\widehat{B}_{0}=\left(\begin{array}{cc}
\frac{1}{2}-K_{0}^{\prime} & \gamma_{n} \operatorname{curl} S_{0} \\
-\gamma_{\times} \nabla S_{0} & \frac{1}{2}+M_{0}
\end{array}\right) .
$$

Here $M_{0}$ is defined by evaluating $n \times \operatorname{curl} S_{0}$ on the boundary. On a smooth boundary, $M_{0}$ is a compact operator in $\mathbf{H}^{-\frac{1}{2}}\left(\operatorname{div}_{\top}, \partial \Omega\right)$. The operators appearing in $(7)$ are well known from the boundary integral equation method for Maxwell's equations [9, Sections 3.3 and 5.5]. For determining $\sigma_{e}\left(\widehat{B}_{0}\right)$, one cannot rely simply on ellipticity in the sense of pseudodifferential operators, which can be determined by computing principal symbols, because of the presence of the space $\mathbf{H}^{-\frac{1}{2}}\left(\operatorname{div}_{\top}, \partial \Omega\right)$. One first has to represent this space by standard Sobolev spaces, for example by Hodge decomposition. We explain some more details in the next section below. The result is $\sigma_{e}\left(\widehat{B}_{0}\right)=\left\{0, \frac{1}{2}, 1\right\}$. We summarize.

Theorem 4.2 If $\partial \Omega$ is smooth, then $\sigma_{e}\left(B_{k}\right)=\left\{0, \frac{1}{2}, 1\right\}$.

Note that although we have obtained $\sigma_{e}\left(A_{k}\right)=\sigma_{e}\left(B_{k}^{0}\right)=\sigma_{e}\left(B_{k}\right)=\left\{0, \frac{1}{2}, 1\right\}$, the corresponding invariant subspaces are quite different for $A_{k}$ and for $B_{k}$, and the commutator of $A_{k}$ and $B_{k}$ is not compact in $\mathbf{H}(\operatorname{curl}, \Omega)$.

\section{The essential spectrum of the complete volume integral operator}

We consider now the operator $\mathcal{A}_{k}^{\eta, \nu}=\eta A_{k}+\nu B_{k}$ on $\mathbf{H}(\operatorname{curl}, \Omega)$. Using (5) and (6), we see that we can write it modulo compact operators as 


$$
\mathcal{A}_{0}^{\eta, \nu}=\nu u(x)+(\eta-\nu) \nabla \int_{\Omega} g_{0}(x-y) \operatorname{div} u(y) d y+(\eta-\nu) \nabla S_{0} \gamma_{n} u(x)+\nu \operatorname{curl} S_{0} \gamma_{\times} u(x) .
$$

Again $\nabla \mathrm{H}_{0}^{1}(\Omega)$ is an invariant subspace on which $\mathcal{A}_{0}^{\eta, \nu} u=\eta u$ and $\mathbf{X}$ is an invariant subspace on which $\mathcal{A}_{0}^{\eta, \nu} u=\nu u-(\eta-\nu) \nabla S_{0} \gamma_{n} u+\nu \operatorname{curl} S_{0} \gamma_{\times} u$. Repeating the arguments that led to (7), we find the spectrally equivalent system of boundary integral equations

$$
\widehat{\mathcal{A}}_{0}^{\eta, \nu}=\left(\begin{array}{cc}
\frac{1}{2}(\nu+\eta)+(\eta-\nu) K_{0}^{\prime} & \nu \gamma_{n} \operatorname{curl} S_{0} \\
(\eta-\nu) \gamma_{\times} \nabla S_{0} & \nu\left(\frac{1}{2}+M_{0}\right)
\end{array}\right) .
$$

Define mappings $T$ from $\mathbf{Y}$ to $\mathbf{Z}=\mathrm{H}^{-\frac{1}{2}}(\Omega) \times \mathrm{H}^{\frac{1}{2}}(\Omega) \times \mathrm{H}^{-\frac{1}{2}}(\Omega)$ and $S$ from $\mathbf{Z}$ to $\mathbf{Y}$ by $T(v, w)=$ $\left(v, V_{0} \operatorname{div}_{\top} w, V_{0} \operatorname{curl}_{\top} w\right), S(v, p, q)=\left((\eta+\nu) v / 2+\nu q,(\nu-\eta) \operatorname{curl}_{\top} V_{0} v-2 \nu \nabla_{\top} V_{0} p+2 \nu \operatorname{curl}_{\top} V_{0} q\right)$. Here $V_{0}$ is the boundary integral operator of the harmonic single layer potential and $\operatorname{curl}_{\top}$ and $\operatorname{curl}_{\top}$ are the scalar and vector surface curls. Then on a smooth boundary $S T-\widehat{\mathcal{A}}_{0}^{\eta, \nu}$ is compact in $\mathbf{Y}$, and $T S$ acts in $\mathbf{Z}$ up to a compact perturbation as the multiplication with the constant matrix

$$
A_{0}^{\eta, \nu}=\left(\begin{array}{ccc}
\frac{\eta+\nu}{2} & 0 & \nu \\
0 & \frac{\nu}{2} & 0 \\
\frac{\nu-\eta}{4} & 0 & \frac{\nu}{2}
\end{array}\right)
$$

Using Lemma 4.1, we see that $\sigma_{e}\left(\widehat{\mathcal{A}}_{0}^{\eta, \nu}\right) \backslash\{0\}$ in $\mathbf{Y}$ is given by the eigenvalues of the matrix $A_{0}^{\eta, \nu}$, which are $\left\{\frac{\eta}{2}, \frac{\nu}{2}, \nu\right\}$. It follows

Theorem 5.1 If $\partial \Omega$ is smooth and $\eta, \nu \in \mathbb{C}$, then for $\mathcal{A}_{k}^{\eta, \nu}=\eta A_{k}+\nu B_{k}$ we have

$$
\sigma_{e}\left(\mathcal{A}_{k}^{\eta, \nu}\right)=\left\{0, \frac{\eta}{2}, \eta, \frac{\nu}{2}, \nu\right\} .
$$

\section{References}

[1] M. M. Botha. Solving the volume integral equations of electromagnetic scattering. Journal of Computational Physics, 218(1):141 - 158, 2006.

[2] N. V. Budko and A. B. Samokhin. Spectrum of the volume integral operator of electromagnetic scattering. SIAM J. Sci. Comput., 28(2):682-700, 2006.

[3] D. Colton and R. Kress. Inverse acoustic and electromagnetic scattering theory, volume 93 of Applied Mathematical Sciences. Springer-Verlag, Berlin, second edition, 1998.

[4] M. Costabel. Some historical remarks on the positivity of boundary integral operators. In Boundary element analysis, volume 29 of Lect. Notes Appl. Comput. Mech., pages 1-27. Springer, Berlin, 2007.

[5] M. Costabel, E. Darrigrand, and E. H. Koné. Volume and surface integral equations for electromagnetic scattering by a dielectric body. J. Comput. Appl. Math., 234(6):1817-1825, 2010.

[6] M. J. Friedman and J. E. Pasciak. Spectral properties for the magnetization integral operator. Math. Comp., 43(168):447-453, 1984.

[7] A. Kirsch. An integral equation approach and the interior transmission problem for Maxwell's equations. Inverse Probl. Imaging, 1(1):159-179, 2007.

[8] A. Kirsch and A. Lechleiter. The operator equations of Lippmann-Schwinger type for acoustic and electromagnetic scattering problems in $L^{2}$. Appl. Anal., 88(6):807-830, 2009.

[9] J.-C. Nédélec. Acoustic and electromagnetic equations, volume 144 of Applied Mathematical Sciences. Springer-Verlag, New York, 2001.

[10] J. Rahola. On the eigenvalues of the volume integral operator of electromagnetic scattering. SIAM J. Sci. Comput., 21(5):1740-1754, 2000. 